\title{
Mapeando o uso de Jogos para o Ensino de Arte-Educação: uma Revisão Sistemática de Literatura e Pesquisa Netnográfica
}

\author{
Rebeca Sasso Laureano, Giliane Bernardi ${ }^{1{ }^{12}}$, Andre Zanki Cordenonsi ${ }^{1{ }^{3}}$ \\ ${ }^{1}$ Programa de Pós-Graduação em Tecnologias Educacionais em Rede - UFSM - rebeca.sasso@gmail.com \\ ${ }^{2}$ Departamento de Computação Aplicada - UFSM - giliane@inf.ufsm.br \\ ${ }^{3}$ Departamento de Arquivologia - UFSM - andrezc@inf.ufsm.br
}

Resumo. O ensino da arte-educação é, atualmente, entrelaçado aos temas contemporâneos tais quais as tecnologias digitais e temas de cultura visual, suas produções, seus sentidos e seus compartilhamentos. Sendo assim, as Tecnologias Educacionais possuem um papel importante como uma possibilidade de modificar e integrar espaços de ensino, atuando na arte-educação como um propulsor de encontros entre estes temas, problematizando a cultura e as maneiras como enxergamos a realidade ao nosso redor. Uma das formas de propor estes encontros são os jogos, presentes no cotidiano de muitos estudantes e que apresentam inúmeras possibilidades didáticas. Neste artigo, propõe-se mapear o uso de jogos para o ensino de arte-educação através de duas abordagens: uma Revisão Sistemática de Literatura e uma Pesquisa Netnográfica realizada em uma rede social. Como resultados, verificou-se que a relação entre a arte-educação e os jogos ainda é incipiente no Brasil, tanto no meio acadêmico, quanto nas práticas pedagógicas dos professores em exercício, alvos da pesquisa netnográfica, indicando que a mesma se apresenta como um campo fértil para a pesquisa.

Palavras-chave: Arte-Educação, Jogos, Revisão Sistemática da Literatura, Pesquisa Netnográfica.

\section{Mapping the Games Use to Art Education Teaching: A Systematic Review of Literature and Netnography Research}

\begin{abstract}
The art education teaching is currently intertwined with contemporary themes such as digital technologies and themes of visual culture, productions, senses and shares. Therefore, Educational Technologies play an important role as a possibility to modify and integrate teaching spaces, acting in art education as an interface booster between these themes, problematizing culture and the ways in which we see the reality around us. The games can be used as an interface between these elements; the games are present in the daily lives of many students and that present numerous didactic possibilities. In this paper, it is proposed a map of the use games for teaching art education through two approaches: a systematic literature review and a netnography research carried out on a social network. As a result, it was found that the relationship between art education and games is still incipient in Brazil, both in the academic environment and in the pedagogical practices of teachers in practice, targets of netnography research, indicating that it presents itself as a fertile field for research.
\end{abstract}

Keywords: Art Education, Games, Systematic Review of Literature, Netnography Research

\section{Introdução}

De acordo com Lopes (2017), a arte é tencionada pelas tecnologias e atua (re)significando os objetos artísticos, influenciando e sendo influenciada através da história. É difícil conceber professores de artes visuais distantes dos meios digitais quando temos como referência estudantes e artistas conectados boa parte do dia, sendo submersos de comunicação instantânea e discursos visuais. Por outro lado, é uma realidade que muitos estudantes, hoje, possuem contato com redes sociais, jogos e outras manifestações visuais quase diariamente.

Huizinga (2007) define o jogo como uma manifestação cultural proveniente de uma ação voluntária e livre, que inclui uma evasão ao mundo real, constituindo um ambiente 
único, porém com total seriedade. Outros pontos podem ser elencados, como o desafio, a interatividade, o ambiente de jogo e os mecanismos de feedback (BOLER e KAPP, 2018). Cada autor, dependendo de suas referências e de seu período histórico, propõe evoluções e atualizações ao conceito, adaptando às mudanças de paradigmas e de comportamento e, neste sentido, a pesquisa em jogos se torna ainda mais potente, pois se trata de uma pesquisa viva, em movimento.

Os jogos têm sido usados na educação, como atividade em si, por meio de atividades na educação básica e como uma possibilidade metodológica de ensino curricular. Muitos autores da educação, como Brougère (1988) e Kishimoto (2008, 2009,2010 ) elaboram seus argumentos no fato de que a educação envolve imaginação e se relaciona ao cotidiano infantil, sendo que, a partir destas relações, a educação pode apropriar-se de elementos do brincar e da ludicidade para compartilhamento de símbolos, tornando as experiências do aprender significativas.

A popularização dos computadores e smartphones e seu fomento pelas políticas públicas no campo da educação digital e inovação em sala de aula, também forneceu aos jogos digitais um espaço de destaque (GEE, 2003). Autores como Gee $(2003,2017)$ e Boller e Kapp (2018) trazem ao debate os conceitos de "bons jogos" e de "jogos eficazes" e de como estes podem conduzir jogadores a soluções criativas para problemas, estimulando a tolerabilidade ao fracasso e o diálogo com situações e imagens relacionadas a realidade. Apesar das diferentes plataformas nas quais são desenvolvidos os jogos, pesquisadores das mais diversas áreas do conhecimento se debruçam em pensar estratégias de ensino, em analisar seus potenciais de aprendizagem, socialização e de como se dão as mediações com as tecnologias.

Sendo assim, é preciso uma pesquisa aprofundada do que se tem produzido na área de jogos e de como este campo é capaz de auxiliar no ensino da arte-educação tendo em vista ser um campo fértil em estudos. Para isto, foi adotada a Revisão Sistemática de Literatura, seguindo abordagem proposta por Kitchenham (2004), para investigar em literaturas científicas da área da Educação, Informática e Artes, como os jogos vêm sendo usados no ensino de artes visuais e, mais especificamente, como estão sendo desenvolvidos. Além disso, esta revisão foi complementada por uma pesquisa netnográfica mista (KOZINETS, 2014), realizada em comunidades/grupos de professores de Artes no Facebook. As próximas seções descrevem os resultados destes levantamentos.

\section{Revisão Sistemática de Literatura}

De acordo com Kitchenham (2004), o primeiro momento de uma revisão sistemática ocorre na elaboração do protocolo e, para tal ação, é necessário inicialmente pontuar os questionamentos da pesquisa. Foram definidas cinco questões de pesquisa para nortear a revisão, sendo que a primeira é considerada a central e as restantes dependem do resultado desta primeira. Por meio dessas perguntas, pode-se evidenciar e levantar discussões acerca do tema e das problemáticas da pesquisa, a fim de respondê-las por meio de pesquisas já elaboradas em outros contextos. As questões selecionadas foram:

QP1: "Que jogos têm sido desenvolvidos no contexto do ensino de artes?

QP2: “As ações de desenvolvimento dos jogos identificados na QP1 envolveram estudantes ou apenas professores ou outros profissionais?

QP3: "Quais os principais objetivos dos jogos identificados na QP1?” 
QP4: "Que metodologias/abordagens de desenvolvimento são utilizadas na elaboração dos jogos identificados na QP1?"

QP5: "Que contribuições são encontradas no desenvolvimento das tecnologias identificadas na QP1?"

QP6: "Os jogos desenvolvidos foram avaliados? Em caso afirmativo, que metodologias/abordagens de avaliação foram empregadas?"

Na continuidade do protocolo, são definidos os critérios de inclusão e exclusão de artigos, responsáveis por orientar a seleção dos estudos primários e isolar apenas artigos de interesse na pesquisa. Nesta pesquisa, tais critérios foram: das Artes

I1: Estudos que apresentam o desenvolvimento de jogos no contexto do ensino

I2: Estudos que apresentam o desenvolvimento de jogos no contexto do ensino interdisciplinar, multidisciplinar ou transdisciplinar, incluindo artes e/ou cultura visual.

E1: Estudos que não apresentam o desenvolvimento de jogos no contexto do ensino das Artes.

E2: Estudos que não apresentem o desenvolvimento de jogos no contexto do ensino interdisciplinar ou transdisciplinar incluindo artes e/ou cultura visual. Brasileiro.

E3: Estudos que não apresentam o desenvolvimento de jogos no contexto

E4: Estudos não disponíveis para leitura digital.

E5: Estudos que sejam dissertações ou teses propriamente ditas.

O desenvolvimento de jogos no contexto interdisciplinar, multidisciplinar ou transdisciplinar foi escolhido pela possibilidade do ensino das artes e da cultura visual estar relacionado a outras disciplinas do ensino fundamental e médio e também do próprio desenvolvimento de jogos.

$\mathrm{Na}$ sequência, são definidas as estratégias de busca, selecionando os locais de pesquisa onde serão realizadas as buscas dos estudos. Para que se obtenha êxito, é necessário estabelecer uma string de busca, ou seja, o conjunto de palavras-chave para localização de estudos dentro do escopo da pesquisa. Neste caso, a string adotada foi:

("jogo" OR "jogos" OR "game” OR "games" OR "jogo educacional” OR "jogos educacionais") AND ("arte" OR "artes" OR "educação artística” OR "Arteeducação" OR "Interdisciplinar" OR "Interdisciplinariedade” OR "Transdisciplinar" OR "Transdisciplinariedade" OR "Multidisciplinar" OR “Multidisciplinariedade")

Foram selecionados estudos do ano de 2015 até o ano de 2019. As fontes de dados incluem os principais eventos e periódicos de Informática, Arte e Educação do Brasil, sendo estes: Anais do Workshop de Informática na Escola (WIE), Simpósio Brasileiro de Informática na Educação (SBIE); Workshops do Congresso Brasileiro de Informática na Educação (WCBIE); Revista Brasileira de Informática na Educação (RBIE); Revista Novas Tecnologias na Educação (RENOTE); Simpósio Brasileiro de Games e Entretenimento Digital (SBGAMES); Portal de Periódicos da Capes (CAPES/MEC); Revista GEARTE (GEARTE); Revista Digital do Laboratório de Artes Visuais (RDLAV); Revista Educação, Artes e Inclusão (REAI); e Revista Educação \& Realidade.

Durante a condução, foram feitas as buscas preliminares, ou seja, a identificação de estudos com as combinações dos termos definidos no planejamento, a seleção dos estudos, avaliação destes estudos, extração de dados e síntese dos dados 
(KITCHENHAM, 2004, p. 8). Nesta etapa foram obtidos 8 resultados nos anais do SBIE, 5 resultados na revista RBIE, 7 resultados nos anais do WCBIE, 14 resultados na revista RBIE, 10 resultados nos anais do SBGames, 5 resultados na revista GEARTE, 3 resultados na revista LAV, 4 resultados na Revista REAI e 7355 resultados para os periódicos da CAPES, totalizando 7411 resultados. Não houve resultados na Revista Educação e Realidade nem nos anais do WIE.

Feita a busca preliminar, foram executadas três seleções. Na primeira seleção, houve a aplicação dos critérios de inclusão, a partir da leitura do título e das palavraschave de cada trabalho, que resultou em um total de 92 artigos mantidos. Na segunda seleção, ocorreu a aplicação dos critérios de exclusão, a partir da leitura da introdução e conclusão, totalizando 29 artigos mantidos e, finalmente, na terceira seleção a leitura completa do artigo, restando apenas 5 artigos que atenderam completamente os objetivos da pesquisa.

\subsection{Análise dos resultados}

Inicialmente, antes da análise, faz-se necessário trazer esclarecimentos sobre o uso do termo "jogos" na área das artes. Em primeiro lugar, principalmente nas áreas do Teatro, das Artes Cênicas e da Psicologia, é muito comum utilizar a palavra "jogo" para referirse ao jogo cênico ou jogo teatral, diverso do conceito de game que procura-se explorar nesta pesquisa. Em segundo, o termo "arte" é muito utilizado no desenvolvimento de jogos e na área da informática e design, sendo este referido a parte estética dos produtos, como layouts, cenários, dentre outros, e não do ensino específico de arte. Em decorrência destas duas observações, a pesquisa em caráter exploratório apresentou um alto número de resultados.

Por meio da análise dos resultados da primeira seleção, foi constatado que existem mais artigos sobre o desenvolvimento de jogos do que artigos sobre metodologias de desenvolvimento de jogos e de análises teóricas, como revisões sistemáticas, avaliações de jogos, relatos de experiência, dentre outros. Quanto a plataformas de desenvolvimento, percebeu-se que, tanto jogos digitais quanto analógicos, são desenvolvidos, porém com uma pequena margem de preferência para $o$ desenvolvimento de jogos digitais.

Também por meio da análise dos dados da segunda revisão, onde ainda não havia se excluído produções que não envolvessem a área das artes, percebeu-se que o ensino inter, trans e multidisciplinar é priorizado quando se trata do desenvolvimento e análise teórica, tendo como principal representante o desenvolvimento de jogos nas áreas de Linguagens, Códigos e suas Tecnologias (39), seguido por Ciências da Natureza, Matemática e suas Tecnologias (30), Educação Inclusiva, Indígena e do Campo (12), Linguagens, Códigos e suas Tecnologias (6) e, por fim, Ciências Humanas e suas Tecnologias (5).

Na terceira seleção, obteve-se quatro relatos de desenvolvimento de jogos e um relato de desenvolvimento de um jogo em nível de protótipo. Foram desenvolvidos dois jogos digitais, uma proposta do desenvolvimento de jogos do tipo Live Action Role Play (LARP), uma proposta de desenvolvimento de jogos na educação infantil e uma proposta de desenvolvimento de jogos analógicos na escola em contexto inclusivo.

Viana, Batista e Lima (2018) apresentam um jogo de perguntas para dispositivos móveis chamado "QuizArt", que apresenta questões sobre história da arte. O jogo foi desenvolvido por profissionais da área da tecnologia e de acordo com os autores possui por objetivo "auxiliar no processo de aprendizagem nos diversos conteúdos do ramos da 
arte" (VIANA, BATISTA E LIMA, 2018, p. 265). A metodologia de desenvolvimento adotada foi a Feature Driven Development (FDD) ou Desenvolvimento Guiado por Funcionalidades, que é utilizada para o desenvolvimento de softwares e inclui processos rigorosos, de modelagem, planejamento prévio e controle do projeto. Ainda, no artigo, não é apresentada uma avaliação do aplicativo junto a professores, apenas que foram consultados e sua aplicação em sala de aula foi citada como uma possibilidade futura.

Martins (2019) apresenta uma proposta diferente. O autor, estudante do curso de Design de Games, propõe para uma escola de ensino integrado, oficinas de desenvolvimento de um jogo de interpretação de papéis do tipo LARP. O objetivo é integrar o conteúdo de oito cursos técnicos ao longo do ano letivo, desenvolvendo jogos do tipo LARP com auxílio dos docentes de seus respectivos cursos. Na descrição da metodologia, consta como se deu a organização dos grupos de desenvolvimento do autor com mais 15 educandos, sendo definidas 6 etapas: contextualizar o projeto aos demais cursos, explicar o que é LARP aos demais cursos, definir o estilo de LARP mais adequado ao perfil dos educandos, produzir as regras do LARP, executar playtest do projeto e coordenar o projeto. Nesse contexto, a prática artística se deu através do universo sensorial do jogo, como elementos de estética, utilizando fotografias, figurinos, cenografia, bem como foram desenvolvidas ilustrações de personagens. Não é definida uma metodologia específica de avaliação, porém, no artigo, consta uma análise dos pontos fortes e fracos da proposta, como a necessidade de comunicação dos organizadores com os grupos de desenvolvimento e também com a escola.

Cassol et al. (2016, p. 990) desenvolveram o jogo "Sete Povos", um jogo para dispositivos móveis, desenvolvido para contar a história dos sete povos das missões jesuíticas no Brasil, que se localiza no Rio Grande do Sul. Além da versão para dispositivos móveis, foi desenvolvida uma experiência imersiva em realidade virtual (RV). É importante salientar que, diferente das outras propostas, este jogo foi desenvolvido por uma equipe interdisciplinar de "historiadores, pesquisadores, game designers, roteiristas, artistas, programadores e também alunos". O objetivo do jogo era desenvolver a aprendizagem de forma natural, especialmente para o ensino de história, buscando o engajamento e a motivação para aprender. A metodologia de desenvolvimento foi descrita não como uma abordagem ou um método específico, mas como ações que levaram ao desenvolvimento do jogo e também do ambiente em RV, sendo elas: uma dinâmica com os desenvolvedores, professores e estudantes para identificar a história do usuário (user story), reunião da equipe de desenvolvimento para conceitualização, insights e brainstorming (chuvas de ideias) a partir dos dados coletados na dinâmica, criação de sketches representando cada conceito, desenvolvimento do jogo propriamente dito, avaliação e playtest monitorado junto aos estudantes de ensino fundamental, onde foram abertas possibilidades para feedback sobre o jogo. Não são especificados, no artigo, se foram usados métodos ou abordagens específicas de avaliação e testagem.

No artigo de Peres e Mastroberti (2017), é narrado um projeto para o desenvolvimento de um jogo aplicado a educação infantil na área das Artes Visuais, apropriando-se da abordagem de Reggio Emilia, desenvolvida no município de mesmo nome (Itália). A proposta tem por objetivo desenvolver um jogo digital por meio das ilustrações das crianças e ainda se encontra em fase de desenvolvimento, sendo que foi feito um protótipo de jogo, chamado "Bicitante" com o material recolhido de outro projeto de Reggio Emilia. A metodologia de desenvolvimento se dá por meio de três fases: a primeira é a compreensão e observação da turma onde será aplicada a proposta, 
a segunda onde são pensados os interesses e curiosidades das crianças e na terceira etapa, onde é proposto o plano de ensino, é realizada a coleta de material (desenhos das crianças para o jogo) e ocorre o desenvolvimento do jogo. Não houve uma metodologia específica para a avaliação do protótipo.

Por fim, Rocha e Silva (2015) apresentam um estudo de caso que ocorre em oficinas do PIBID (Programa Institucional de Bolsas de Iniciação à Docência) na área de educação inclusiva e interdisciplinar, por meio do desenvolvimento de jogos interdisciplinares e inclusivos. Participaram das atividades estudantes de artes visuais, música, pedagogia, história e geografia. Foram desenvolvidos 11 jogos analógicos com grupos específicos de estudantes. A metodologia adotada foi a de Fonseca da Silva, Mendes e Schambeck (2012), iniciando pela coleta de dados, como necessidades, materiais e projetos; produção e estudo dos materiais e propostas considerando os professores específicos e, por último, a experimentação dos projetos e a criação final dos jogos. A avaliação se deu por meio de testes de jogabilidade com os próprios estudantes e também por meio de análise qualitativa, levando em consideração as informações gráficas, as mecânicas e também a qualidade dos materiais.

É interessante observar que nas propostas de Peres e Mastroberti (2017); Martins, (2019) e Rocha e Silva (2015) as artes visuais surgem como uma proposta do desenvolvimento de materiais para o jogo, como ilustrações, desenhos, arte para o tabuleiro ou para os elementos do game, enquanto que nos jogos Sete Povos e QuizArt as artes visuais surgem relacionadas a fatos históricos e/ou culturais, como por exemplo, a história dos sete povos das missões. Percebe-se, então, duas possibilidades para o desenvolvimento e uso de jogos em artes visuais: em primeiro, o desenvolvimento de materiais gráficos junto aos estudantes e em segundo, o estudo de temas culturais que atravessam a narrativa do jogo. Foi possível observar também a importância do diálogo com professores e estudantes antes do desenvolvimento do jogo, pois apenas um jogo foi desenvolvido antes de ser apresentado aos professores e estudantes da área. O ensino interdisciplinar e inclusivo também se fez bastante presente nesta revisão, evidenciando ser uma demanda ativa e também uma possibilidade para o desenvolvimento de jogos.

Sobre as plataformas de desenvolvimento, duas das pesquisas apresentadas inserem-se em um contexto analógico, porém utilizando tecnologias em algum momento de seu desenvolvimento (como máquina fotográfica, computador ou celular). Na pesquisa de Rocha e Silva (2015) e de Peres e Mastroberti (2017), as tecnologias surgem como possibilidades na área gráfica de desenvolvimento (digitalização, desenvolvimento de ilustrações, etc.) enquanto que na de Martins (2019) as tecnologias surgem como recurso adicional para construção de narrativas (fotografia de cenários, ilustrações. etc.).

Quanto a avaliação destes jogos e das atividades, dois dos artigos não apresentam nenhum dado sobre avaliação ou testagem pelos estudantes, sendo estes descritos como possibilidades futuras. Nos outros três artigos de Viana, Batista e Lima (2018); Cassol et al. (2016) e Rocha e Silva (2015) a avaliação não se dá por meio de uma metodologia específica, sendo elas qualitativas por meio de feedbacks e diálogos com os estudantes a respeito, por exemplo, dos gráficos, da jogabilidade e da qualidade do jogo.

Também é importante ressaltar que nenhum dos artigos propõe passos ou atividades descritas para que outros professores possam desenvolver seus jogos em sala de aula ou com outras equipes, sendo ainda necessário conhecimentos específicos de game design, programação, dentre outras áreas do conhecimento, que, muitas vezes, não são explicadas ou mencionadas durante os relatos de experiência. 


\section{Estudo Netnográfico: Jogos, Professores de Artes e Facebook}

Considerando o analisado na seção anterior, pode-se inferir que os jogos nas Artes Visuais ocorrem por meio de propostas interdisciplinares e também por meio de propostas que visam currículos específicos, como a história da arte, sendo estes estudos publicados por pesquisadores de diversas áreas. No entanto, muitas vezes, as pesquisas acadêmicas se distanciam da realidade escolar, permanecendo restrita aos locais de aplicação das propostas. Visando contribuir para o diálogo entre universidade e contexto escolar, buscou-se outras maneiras de mapear a construção e o desenvolvimento de jogos que não fosse apenas por meio de produções acadêmicas, mas também pelo relato dos próprios professores de artes.

Desta forma, foi realizada uma pesquisa Netnográfica Mista, onde foram observados alguns pontos da Netnografia (KOZINETS, 2014), sem se ater a todas diretrizes propostas. A investigação se deu por meio da rede social Facebook com foco no público de professores de artes, em específico, brasileiros. A partir dos mecanismos de busca encontrados dentro da rede, foi solicitada entrada nos seguintes grupos da rede social: "Professores de Artes" , "Professores de Artes do Brasil"2 e "Sou professor de Artes"3. Nestes grupos, há professores com formação específica em Artes Visuais, Música, Dança ou Teatro, assim como professores sem licenciatura em Artes, mas que atuam na área, realidade bastante expressiva no Brasil. A pesquisa ocorreu no segundo semestre de 2019.

Para esta pesquisa, foram realizados três tipos de coletas de dados propostos por Kozinets (2014): dados arquivais, dados extraídos e dados de notas de campo. No primeiro caso, foram selecionadas postagens tais como foram publicadas; no segundo, dados extraídos pelo pesquisador por meio de interações e, no terceiro, as anotações e avaliações de acordo com suas experiências e histórias. Inicialmente, foi informado ao grupo que a pesquisa estava inserida no contexto da Universidade <omitido_para_revisão>, na área de Tecnologias Educacionais, em especial envolvendo o uso de jogos no âmbito escolar. Após a apresentação, por meio da ferramenta de busca, foi feita a busca do termo 'jogo' dentro de cada grupo.

Os grupos serão referenciados como Grupo A (6827 membros, criado em 2014), Grupo B (7885 membros, criado em 2014) e Grupo C (10342 membros, criado em 2016). Como as publicações no Facebook costumam ser mais numerosas e também mais instáveis quanto a sua permanência, foram selecionadas publicações de janeiro de 2017 até dezembro de 2019. Também é importante salientar que postagens que se referiam a jogos teatrais, como já relatado, foram ignoradas. No Grupo A foram encontradas 7 publicações: 1 anúncio de venda de um jogo comercial adaptado com currículo da história da arte; 1 anúncio de venda de jogo da memória com obras de Rembrandt; 1 anúncio de tabuleiro com tema de artistas modernistas; 1 anúncio de Workshop de Jogos e Brincadeiras em contexto de educação emocional; 1 sugestão de um membro para a Apostila de jogos com obras de Picasso (à venda); 1 anúncio de venda de jogo de cartas do tipo 'trunfo'; 1 anúncio de venda de jogo de cartas sobre pinturas e pintores e 1 anúncio de venda de apostila com um jogo de blocos. Todos os anúncios de venda de jogos foram feitos pelo mesmo membro.

\footnotetext{
${ }^{1}$ https://www.facebook.com/groups/1414945025438085/

2 https://www.facebook.com/groups/742399102481986/

3 https://www.facebook.com/groups/673654052798636/ 
No grupo B foram encontradas 6 publicações: 1 anúncio de venda de apostila com 6 jogos de artes para desenvolver e aplicar critérios relevantes na análise de obras; 1 anúncio de venda de apostila de jogos musicais; 1 foto sobre a importância do uso de jogos na educação; 1 vídeo sobre oficina de um jogo analógico; 1 anúncio de apostilas de um jogo de blocos; 1 anúncio de serviço terceirizado com indicações de compras de jogos.

No grupo $\mathrm{C}$ foram encontradas 14 publicações do tipo: 1 anúncio de venda de jogo de cartas sobre tendências e movimentos da arte; 1 uma dúvida sobre sugestões de jogos gratuitos para estudantes que terminam rápido suas atividades; 1 sugestão de jogo digital chamado 'Missão Curupira' para trabalhar o conteúdo de folclore; 1 sugestão de jogo digital 'Interland' do Google; 1 relato de experiência da construção colaborativa de um jogo interdisciplinar (artes, história e geografia) de tabuleiro sobre arquitetura, patrimônio e cultura, em um $5^{\circ}$ ano de uma escola; 1 anúncio de venda de jogo com emojis; 1 relato de experiência de atividade inspirado no jogo "Minecraft"; 1 relato de experiência da construção de jogos tradicionais africanos; 1 relato de uso de jogo da memória com obras de arte; 1 sugestão de jogo para trabalhar artes a partir do quarto ano do Ensino Fundamental; 1 divulgação de jogo autoral para download gratuito; 1 pedido de sugestão de jogos adaptados para aulas de arte; 1 vídeo e anúncio de um jogo sobre arte barroca; 1 anúncio de trunfo de cores.

Por meio de um diário de campo, foram anotadas algumas observações quanto aos grupos e aos membros que publicaram tanto seus relatos como seus produtos. Inicialmente, pode-se perceber que grande parte do volume das postagens refere-se a anúncios de vendas de jogos, desenvolvidos por um único professor que compartilha em todos os grupos do Facebook o seu trabalho. Ele relata o sucesso da proposta, o que o levou a comercializar os jogos. Ele publicou, no Grupo C, um vídeo jogando com seus estudantes. Esta publicação apresentou 106 curtidas e 168 comentários, sendo a maioria dos comentários demonstrava interesse no produto. Em um dos comentários, quando questionado sobre as motivações da comercialização de jogos, o autor da publicação compartilha que a produção foi motivada pela ausência de materiais do tipo na área. Não houve discussão, no entanto, sobre a possibilidade dos professores construírem seus próprios jogos.

O Grupo $\mathrm{C}$ foi o que apresentou maior número de interações e publicações (14) envolvendo jogos. Além disso, as postagens apresentavam mais comentários e curtidas em comparação entre os grupos A e B. Houve duas postagens onde professores apresentavam jogos criados por eles: um jogo de memória, disponibilizado gratuitamente (110 comentários, 53 curtidas e 3 compartilhamentos); e outra sobre a criação colaborativa de um jogo com os estudantes sobre patrimônio (193 curtidas e 36 comentários). Ambas postagens são bastante significativas em comparação a outras publicações selecionadas pelo termo de pesquisa 'jogos' neste grupo. Os comentários incluíram pedidos dos planos de aula e questionamentos sobre materiais. Algumas professoras compartilharam seus planos de aula envolvendo jogos, incluindo até mesmo uma foto de um jogo feito no formato de 'trilha'.

Em contato com os autores do Jogo do Patrimônio, os autores narraram a importância de o professor buscar um ensino que seja agradável ao entendimento do estudante, diferente, muitas vezes, da maneira como o professor aprendeu. Os autores também informaram que já está em fase de desenvolvimento outro jogo, desta vez com conexões entre matemática, artes e física. Em específico, este projeto sobre patrimônio, foi tema de uma reportagem chamada "Jogo de tabuleiro aproxima estudantes de 
patrimônio histórico em SC" onde é narrado o processo de desenvolvimento e de continuidade da ação junto a comunidade na qual atua (VIEIRA, 2019). Por outro lado, um dos pedidos de sugestões de jogos no Grupo $C$ não foi respondido, apenas curtido por um membro. Isso demostra que, apesar dos professores se interessarem pelo tema de jogos e também reagirem positivamente em relatos de experiências, não há interações em publicações com sugestões de jogos ou pedidos de sugestões, o que parece indicar um desconhecimento e ou um baixo número de jogos direcionados para a arte-educação.

Por fim, é importante perceber que foram encontradas apenas duas sugestões de jogos digitais, que praticamente não teve interações ( 3 curtidas e nenhum comentário; 9 curtidas e 1 comentário), enquanto que o restante das postagens e dos relatos se referem a jogos analógicos, inclusive o inspirado em um jogo digital (Minecraft). Isto vai ao encontro com o problema apresentado nesta pesquisa, que infere que, muitas vezes, por desconhecimento tecnológico, falta de infraestrutura ou pela ausência de planos ou propostas envolvendo jogos digitais, os professores acabam preferindo desenvolver jogos analógicos. Ao mesmo tempo, é bastante claro que os professores compartilham da necessidade de práticas inovadoras, porém, relatam dificuldade em encontrar recursos ou planos de aula na área, visto que a maioria das pessoas que desenvolvem tais materiais não compartilham na íntegra o processo de desenvolvimento de forma instrucional, o jogo ou si de forma gratuita ou opta por fazê-lo apenas comercialmente.

\section{Considerações Finais}

Por meio desta pesquisa, procurou-se encontrar diálogos entre o desenvolvimento e uso de jogos e o ensino de Artes no contexto escolar, buscando investigar como esta tecnologia está sendo empregada nos espaços educativos, considerando o cenário nacional. Elencando algumas bases de dados específicas, envolvendo periódicos e anais de eventos relacionados a Tecnologias e Artes foi possível observar o baixo número de produções voltadas especificamente para o tema.

Um aspecto central que foi buscado, a partir das questões norteadoras da RSL, envolvia identificar metodologias para o desenvolvimento e avaliação de jogos no contexto do ensino de artes, que não retornou resultados. Por mais que as pesquisas envolveram o desenvolvimento, não se identificou abordagens ou metodologias para guiar um processo de produção de jogos para apoiar o ensino de artes. Ainda, investigou-se o caráter colaborativo das propostas, buscando identificar o grau de participação de professores e estudantes no desenvolvimento dos jogos, o que foi considerado relevante por muitos autores, destacando o caráter interdisciplinar envolvido

Por outro lado, frente aos resultados da netnografia, percebe-se que os professores têm interesse em novas metodologias para a arte-educação e, principalmente, no uso de jogos. Exemplos práticos do desenvolvimento, uso e planos de aula utilizando jogos sempre receberam bastante atenção nos grupos analisados. E é bastante pertinente perceber que, sem uma preocupação maior por parte da academia - como evidenciado na RSL -, os professores têm desenvolvido os seus próprios jogos, criando e ou adaptando a partir de títulos já existentes. Esta produção empírica é um sinal claro do interesse dos educadores em exercício na utilização de jogos para a arte-educação.

Desta forma, acredita-se que esta integração, entre tecnologia e professor-arteestudante, atuando no desenvolvimento colaborativo de jogos, pode impulsionar os estudantes à pesquisa e, consequentemente, a aprendizagem dos conteúdos envolvidos. 
Porém, para que isso ocorra de forma satisfatória, apoiando efetivamente os processos de ensino e aprendizagem, é necessária uma formalização das etapas de produção, no que concerne aos aspectos pedagógicos e de caráter técnico, visando apoiar o professor no decorrer desta caminhada. Ao constatar que tais percursos/metodologias não foram encontradas nos resultados desta pesquisa, fica evidenciada a relevância de uma proposta de abordagem para o desenvolvimento de jogos que envolva a produção colaborativa de jogos para o ensino de arte-educação, trazendo etapas a serem seguidas, projeto este que se vislumbra como perspectiva futura ao término desta etapa e que já encontra-se em desenvolvimento no formato de um modelo de unidade de estudo, seguindo as diretrizes propostas por Filatro (2010) para o design instrucional e o modelo proposto por Boler e Kapp (2018) para o desenvolvimento de jogos para aprendizagem.

\section{Referências}

BOLER, S.; KAPP, K. Jogar para aprender: tudo o que você precisa saber sobre o design de jogos de aprendizagem eficazes. São Paulo: DVS Editora, 2018.

BROUGÉRE, Gilles. Jogo e Educação. Porto Alegre: Artes Médicas, 1998.

CASSOL, V. J.; ROSSA, P.; BITTENCOURT, J. R.; MARSON, F.; RIGO, S. J. Sete Povos: Games e Realidade Virtual para contar a história das Missões Jesuíticas no Brasil. In: XV Simpósio Brasileiro de Games, 2016, São Paulo.

FONSECA DA SILVA, M. C. R.; MENDES, R. F.; SHAMBECK, G. M. L. Objetos pedagógicos: uma experiência inclusiva em oficina de artes. Araraquara, SP: Junqueira \& Marin, 2012.

GEE, J. P. What video games have to teach us about learning and literacy. Nova York: Palgrave Macmillan, 2004.

HUIZINGA, J. Homo Ludens: O jogo como elemento de cultura. São Paulo: Editora Perspectiva, 2001. $5^{\mathrm{a}}$ ed.

KISHIMOTO, T. M. (org.) Jogo, brinquedo, brincadeira e educação. São Paulo: Cortez, 2008.

Vozes, 2009. , Jogos Infantis: O jogo, a criança e a educação. 15a . ed. Petrópolis: Ed.

, Brinquedos e brincadeiras na educação infantil. In: Seminário Nacional: Currículo em Movimento - Perspectivas Atuais, 1., 2010, Belo Horizonte.

KITCHENHAM, B. Guidelines for performing Systematic Literature Reviews in Software Engineering. EBSE Technical Report. Durham: University of Durham. 2007.

KOZINETS, R. V. Netnografia: Realizando pesquisa etnográfica online. Porto Alegre: Penso, 2014.

LOPES, F. A. S. Mídia, arte e tecnologia: uma reflexão contemporânea. Comunicação e Sociedade, Braga, v.31, p. 287-298, jun. 2017.

MARTINS, D. S. Último Recurso: Uma experiência de LARP Multidisciplinar. In: XV Simpósio Brasileiro de Games, 2016, São Paulo.

PERES, C.; MASTROBERTI, P.; O Jogo Digital como Proposição Educativa: experiências ludopoéticas na infância. In: XVI Simpósio Brasileiro de Games, 2017, São Paulo. p. 343-345.

ROCHA, S. R. P.; SILVA, M. C. R. F. Pibid Interdisciplinar: Um Olhar sobre as Contribuições da Arte para a Construção de Objetos Pedagógicos para Pessoas com Deficiências. Rev. Educação Artes e Inclusão, Florianópolis, v.11, n.1, 2015.

VIANA, L.; BATISTA, E. J. S.; LIMA, A. C. QuizArt: Um aplicativo para auxiliar o Ensino e a Aprendizagem de Arte. In: VII Congresso Brasileiro de Informática na Educação, 2018, Fortaleza. 